\title{
Unilateral Subthalamic Nucleus Stimulation in the Treatment of Asymmetric Parkinson's Disease with Early Motor Complications
}

\author{
Michał SOBSTYL ${ }^{1}$, Mirosław ZABEK ${ }^{1}$, Artur ZACZYNSKI ${ }^{2}$, Wojciech GORECKI ${ }^{2}$, Zbigniew MOSSAKOWSKI ${ }^{2}$, \\ Grażyna BRZUSZKIEWICZ-KUZMICKA ${ }^{3}$ \\ ${ }^{1}$ Postgraduate Medical Center, Department of Neurosurgery, Warsaw, Poland \\ ${ }^{2}$ Bródno Regional Hospital, Department of Neurosurgery, Warsaw, Poland \\ ${ }^{3}$ Józef Piłsudski University of Physical Education, Faculty of Rehabilitation, Warsaw, Poland
}

\section{ABSTRACT}

AIM: The aim of this study was to assess the results of unilateral subthalamic nucleus (STN) stimulation for the treatment of Parkinson's disease (PD) with marked asymmetry of parkinsonian motor symptoms and early motor complications.

MATERIAL and METHODS: The clinical series consisted of 32 consecutive PD patients, in whom unilateral STN stimulation was performed. All patients were assessed according to the Unified Parkinson's Disease Rating Scale (UPDRS), and Hoehn and Yahr staging. The patients were assessed preoperatively, and at 12, and 24 months after unilateral STN stimulation. 22 patients were followed for 2 years.

RESULTS: Medication off/stimulation on total UPDRS motor scores were improved by $29 \%$ when compared to the baseline medication off motor scores. The contralateral motor scores improved by $49 \%$, whereas the axial motor scores by $18 \%$ in medication off/stimulation on condition. The duration and severity of levodopa induced dyskinesia were reduced respectively by $73 \%$ and by $77 \%$. The daily levodopa dose was decreased by only $10 \%$.

CONCLUSION: Unilateral STN stimulation is a safe and effective procedure for selected patients with marked asymmetry Parkinson's disease motor symptoms and early motor complications.

KEYWORDS: Deep brain stimulation, Unilateral subthalamic nucleus stimulation, Neuronavigation, Stereotactic neurosurgery

\section{INTRODUCTION}

$\mathrm{P}$ arkinson's disease $(P D)$ is diagnosed clinically based on the cardinal motor symptoms including resting tremor, rigidity and bradykinesia. A characteristic feature of early idiopathic PD is asymmetric motor symptom onset. In advanced PD, it is sometimes difficult to recognize which side of the body was first affected by disease. Generally, patients with advanced PD, with severe bilateral levodopa-induced dyskinesia (LID) and motor fluctuations require bilateral subthalamic nucleus (STN) deep brain stimulation (DBS) $(17,21)$.
Patients with advanced PD and older age at surgery may not easily tolerate bilateral STN DBS, unlike younger patients with moderate PD. Moreover, approximately $30 \%$ of patients with long-standing PD develop depression, which is partially attributable to the disease itself. Dementia in PD patients is related to the age of PD onset, rather than to the duration of the disease $(6,9,14,16)$. Psychiatric symptoms - such as compulsive behavior, gambling etc. - may be triggered or aggravated by long-term medication, especially in dopamine agonist therapy $(14,27)$. Moreover, in the course of the disease a number of 
features that are unresponsive to medication begin to dominate. Nevertheless, pharmacological therapy remains the mainstay treatment for patients affected by PD. Neurosurgical procedures are indicated when pharmacotherapy inadequately controls motor symptoms and LID develop (12).

Generally, patients with shorter PD duration demonstrate better cognitive performance, and are in better overall health at the time of surgery. Moreover, unilateral STN stimulation procedures are less invasive than bilateral simultaneous STN procedures $(2,4,5,8,17,19,22,23)$. Bearing in mind all of the above facts, we aimed to operate on PD patients with the asymmetry of PD symptoms.

\section{MATERIAL and METHODS}

The study included 32 patients (12 female and 20 male) with asymmetric PD, with mild or moderate disability of LID. The patients' demographic and clinical characteristics are presented in Table I. The patients were followed up for either 1 or 2 years (27 and 22 patients, respectively). The specific reasons for dropout at 2 years' follow-up were as follows: 4 patients were lost for follow-up, 3 patients were unwilling to report at 2 years follow-up although they reported clinical improvement but were not assessed using study protocol, in 2 patients stimulation settings were adjusted at other centers, located nearer their place of residence, and 1 patient was diagnosed with metastatic breast carcinoma.

All patients underwent unilateral STN DBS at the Neurosurgical Department of Postgraduate Medical Center, starting from May 2006 till February 2012. Eligibility for the surgery was based on an assessment of the motor symptoms of PD based on the CAPSIT-PD criteria (1). All patients met the UK Brain Bank criteria for the diagnosis of PD and suffered from disabling asymmetric motor symptoms in the off-medication state, including tremor and mild or moderate unilateral LID in the on-medication state. The inclusion criteria were as follows: idiopathic PD confirmed by a movement disorder neurologist; PD continuing for more than 5 years after the diagnosis; the presence of disabling mild or moderate LID rated maximally 2 scores on item 33 of UPDRS part IV. PD was considered asymmetric when motor scores for the more affected side of the body were at least two times higher than for the less affected side. An L-dopa challenge was administered to confirm drug responsiveness according to UPDRS part III. The patients qualified for STN DBS had the improvement of over $33 \%$ of PD motor symptoms when compared to the off state. The offmedication state was defined as an overnight period free of levodopa-containing drugs. Patients with symmetric PD who qualified for staged bilateral STN DBS were excluded from the study. The cognition of the patients was assessed by the Mini Mental State Examination (MMSE). Patients with more than 25 points on the MMSE were considered eligible for surgery. The study received the Institutional Review Board Approval and local Ethic Committee. The patients were informed about possible surgical complications associated with stereotactic surgery and hardware implantation. Coagulopathies were considered as a contraindication to stereotactic surgery. All aspirin-containing medications were withheld at least 14 days before the planned surgery.
The primary outcome measure included the motor part of the UPDRS. Separate motor subscores were assessed for contralateral as well cumulative ipsilateral motor UPDRS subscores. The contralateral motor subscore was the sum of motor scores from limb tremor (items 20-21; range 0-16), limb rigidity (item 22; range 0-8), limb bradykinesia (items 23-26; range $0-36$ ). The axial motor subscore constituted the sum of the following scores for arising from chair, posture, gait, postural instability and body bradykinesia (items 27-31; range 0-20). Separate motor subscores for limb tremor, limb rigidity, limb bradykinesia were also assessed contralaterally in the off medication state preoperatively.

The secondary outcome measure included an assessment of mentation, behavior, and mood (part I of the UPDRS), the activities of daily living (part II of the UPDRS), and complications of therapy (part IV of the UPDRS). Postoperative evaluations were performed at 1 and 2 years after the surgery. All parts of UPDRS were assessed in the off-medication and on-medication states, with the stimulator turned on postoperatively. The results were compared to corresponding preoperative scores in the off-medication and on-medication states, respectively. Clinical assessment also involved the Hoehn \& Yahr staging scale. All patients were evaluated using the Mini Mental State Examination. All patients were assessed in the off-medication and on-medication states prior to surgery. The effects of unilateral STN DBS on UPDRS part I, II, III, and IV, H/Y stage in medication off and on conditions were assessed using a paired $t$-test, based on a normal distribution of values. A level of $p<0.05$ was considered significant.

In brief, the STN was selected as the stereotactic target contralateral to the more affected body side. All the procedures were performed under local anesthesia, and stereotactic STN planning was performed with a neuronavigation device (Stealth Station, Medtronic, Minneapolis) using the Framelink 4 software and intraoperative macrostimulation. To perform intraoperative macrostimulation, the Leksell Neurogenerator (Stereotactic Instruments, Elekta, Stockholm, Sweden) and Leksell macroelectrode with a non-insulated electrode tip of $2 \mathrm{~mm}$ in length and $1.5 \mathrm{~mm}$ in diameter were used. After the STN was located, the electrode (Model 3389, Medtronic, Minneapolis) for chronic stimulation was implanted. The depth of the inserted chronic electrode was controlled by lateral fluoroscopic images. Immediately after the implantation of the DBS lead the skin was sutured and the patients were brought to the computed tomography (CT) stereotactic scanning. Thereafter the patients were introduced into the general anesthesia. Internal pulse generators (Soletra or Activa SC, Medtronic, Minneapolis) were placed in the subclavicular region and connected by means of a connecting wire to the DBS lead, according to the manufacturer's guidelines. All contacts of the implanted DBS lead were checked for impedance (25).

\section{- RESULTS}

22 PD patients benefited from unilateral STN DBS, as determined at 2-year follow-up. Tables II and III show the effect of unilateral STN DBS on parts I, II and III and IV of the UPDRS 
in the off and on state respectively while the stimulators were switched on. The stimulation effect was seen in the off state with a reduction of motor scores by $31 \%$ and $29 \%$ at 1 and 2 years after surgery. Contralateral tremor was reduced by $79 \%$ and $76 \%$ at 1 and 2 years, respectively. Contralateral rigidity was reduced by $43 \%$ and $33 \%$ during subsequent follow-up visits. Contralateral bradykinesia was found to have improved by $52 \%$ and $45 \%$ at 1 and 2 years after surgery.

The effect of unilateral STN DBS on the motor scores in the on state was still evident, reducing motor UPDRS scores by $21 \%$ and $10 \%$ at 1 and 2 years after surgery. This effect can be attributed to unilateral STN DBS and the slightly modified daily levodopa dose during the postoperative period. Unilateral STN DBS contributed to the reduction of duration and disability of LID. The reduction of LID duration was $67 \%$ and $73 \%$ at 1 and 2 years after surgery. The LID disability decreased by $69 \%$ and $77 \%$ at 1 and 2 year follow-up, respectively. This antidyskinetic effect could be only partially attributable to a $10 \%$ reduction of daily levodopa dosage 2 years after surgery. The preoperative MMSE was $27.3+/-2.5$. The postoperative MMSE scores were unchanged after surgery and scored $27.1+/-3.2$ and $26.8+/-2.8$ at 1 and 2 years follow-up. The preoperative and postoperative scores of UPDRS part I before and after the surgery are presented in Tables II and III.

The mean preoperative daily dose of levodopa was $890+/-$ $240 \mathrm{mg}$, as compared to $800+/-2601$ year after unilateral STN DBS and $800+/-190$, and 2 years after unilateral STN DBS.

There was 1 case of intracerebral hemorrhage seen on a postoperative CT scan. In this case, postoperative CT showed minor intraparenchymal bleeding beneath the cortical surface. This bleeding was clinically silent. 1 patient developed transient confusion in the immediate postoperative period.
This patient made full recovery at the time of discharge from the hospital. There was one hardware-related complication (one instance of breakage of the connecting cable). We noted two cases of skin erosion with a subsequent infection over the connector located in the retromastoid region. The patients were reoperated on with a translocation of the connector to the parietooccipital region and firm suturing to the galea. The patients made full recovery, while subsequent antibiotic therapy solved the infection problem with good wound healing. There were no other complications related to the implanted hardware in the 2-year follow-up period.

At 2-year follow-up, a total of 15 patients used monopolar cathodal stimulation, 4 patients used 2 adjacent contacts as cathodes, and 3 patients used bipolar stimulation mode. The stimulating parameters did not change significantly over the follow-up period. During follow-up visits all contacts were screened to measure the range of resistance. Most patients with monopolar stimulation experienced transient paresthesia after switching on the IPGs, which resolved completely after a few seconds. In only three patients bipolar stimulation was used 2 years after surgery. The reason for it was dysartria induced by the monopolar stimulation mode.

\section{DISCUSSION}

Our results confirm high efficacy of unilateral STN DBS in the treatment of asymmetric PD with mild or moderate LID. Our observations are consistent with the results obtained by other investigators who reported a reduction of total motor UPDRS after unilateral STN DBS between 25\% and $43 \%(8,19,22,24,26)$. Unilateral STN DBS is usually applied to patients with moderate PD and total preoperative motor UPDRS score varying between 32 to 45 points in most series $(19,22,24,26)$. In our 32 patients, the total preoperative motor

Table I: Demographic and Clinical Description of 32 Consecutive Patients After Unilateral STN DBS

\begin{tabular}{|c|c|}
\hline Mean age of disease onset (diagnosis) & 48.9 (yrs) range (39.7 to 62.1 yrs) \\
\hline
\end{tabular}

$\begin{array}{lr}\text { Sex of operated } & \\ \text { Patients } & 12 \\ \text { Female } & 20 \\ \text { Male } & 32 \\ \text { Total } & 32\end{array}$

Mean disease duration till unilateral STN DBS 7.2 yrs (range from 5.5 to $10.1 \mathrm{yrs}$ )

Mean age at the surgery 55.6 yrs (range from 45.5 to 72.1 yrs)

Mean preoperative levodopa dose

$890+/-240 \mathrm{mg}$

Laterality of symptoms/asymmetrical disease

Right side dominant

Left side dominant

22

Total

12

32

Side of STN DBS surgery

Right STN DBS

Left STN DBS

yrs: Years, STN: Subthalamic nucleus, DBS: Deep brain stimulation. 
Table II: The Preoperative (Baseline) and Follow-Up Examinations of UPDRS Scores were Performed in Medication off Condition Preoperatively and Postoperatively During Unilateral STN Stimulation (Stimulation on Condition). The Effects of Unilateral STN DBS on Mentation, Behavior, and Mood (part I UPDRS), the Activity of Daily Living Score (ADL part II UPDRS), Motor and Motor Subscores for Contralateral and Cumulative Motor Score for Ipsilateral Motor Features (part III UPDRS), and the Hoehn and Yahr Staging were Analyzed Using a Paired t-Test. A Level of $\mathrm{P}<0.05$ was Considered Significant. For all Scores of UPDRS and H/Y Staging Reduction Means Functional Improvement

\begin{tabular}{lcccc}
\hline Items of UPDRS & Score range & $\begin{array}{c}\text { Baseline scores in } \\
\text { medication off } \\
\text { state }\end{array}$ & $\begin{array}{c}\text { Stimulation on/ } \\
\text { medication off } \\
\text { state 12 months }\end{array}$ & $\begin{array}{c}\text { Stimulation on/ } \\
\text { medication off } \\
\text { state 24 months }\end{array}$ \\
\hline UPDRS Part I & $0-16$ & $3.5+/-1.4$ & $3.0+/-1.2(\mathrm{~ns})$ & $3.5+/-1.0(\mathrm{~ns})$ \\
\hline UPDRS Part II & $0-52$ & $16.7+/-3.4$ & $11.7+/-2.1(\mathrm{~ns})$ & $12.5+/-2.8(\mathrm{~ns})$ \\
\hline UPDRS Part III & $0-108$ & $32.9+/-4.7$ & $22.7+/-3.2\left(^{*}\right)$ & $23.4+/-4.1\left(^{*}\right)$ \\
\hline Contralateral motor features & $0-36$ & $14.6+/-3.9\left(^{*}\right)$ & $6.7+/-3.2\left(^{*}\right)$ & $7.4+/-2.9\left(^{*}\right)$ \\
\hline Ipsilateral motor features & $0-36$ & $7.2+/-2.1$ & $6.3+/-1.5(\mathrm{~ns})$ & $7.5+/-2.3(\mathrm{~ns})$ \\
\hline Axial motor features & $0-20$ & $9.6+/-1.9$ & $7.2+/-0.9\left(^{*}\right)$ & $7.9+/-3.2(\mathrm{~ns})$ \\
\hline Contralateral tremor & $0-12$ & $3.8+/-0.7$ & $0.8+/-0.5\left(^{*}\right)$ & $0.9+/-0.6\left(^{*}\right)$ \\
\hline Contralateral rigidity & $0-8$ & $3.9+/-0.6$ & $2.2+/-0.7\left(^{*}\right)$ & $2.6+/-0.8\left(^{*}\right)$ \\
\hline Contralateral bradykinesia & $0-16$ & $6.9+/-1.2$ & $3.3+/-1.2\left(^{*}\right)$ & $3.8+/-1.0\left(^{*}\right)$ \\
\hline Hoehn and Yahr staging & $0-5$ & $2.2+/-0.6$ & $1.7+/-0.3(\mathrm{~ns})$ & $1.8+/-0.4(\mathrm{~ns})$ \\
\hline ADL Activitis
\end{tabular}

ADL: Activities of daily living, STN: Subthalamic nucleus, UPDRS: Unified Parkinson's Disease Rating Scale, DBS: Deep brain stimulation, (*): Statistical significance, $n s:$ Not significant.

Table III: The Preoperative (Baseline) and Follow-up Examinations of UPDRS Scores were Performed in Medication on Condition Preoperatively and Postoperatively During Unilateral STN Stimulation (Stimulation on Condition). The Effects of Unilateral STN DBS on Mentation, Behavior, and Mood (Part I UPDRS), The Activity of Daily Living Score (ADL Part II UPDRS), Motor and Motor Subscores for Contralateral and Ipsilateral Motor Features (Part III UPDRS), and the Hoehn and Yahr Staging were Analyzed Using a Paired t-Test. A Level of $\mathrm{P}<0.05$ was Considered Significant. Moreover, the Side Effects of Long-Term Levodopa Intake were Analyzed Using the Items Assessing the Severity and Duration of LID from (part IV of UPDRS). For all Scores of UPDRS and H/Y Staging Reduction Means Functional Improvement

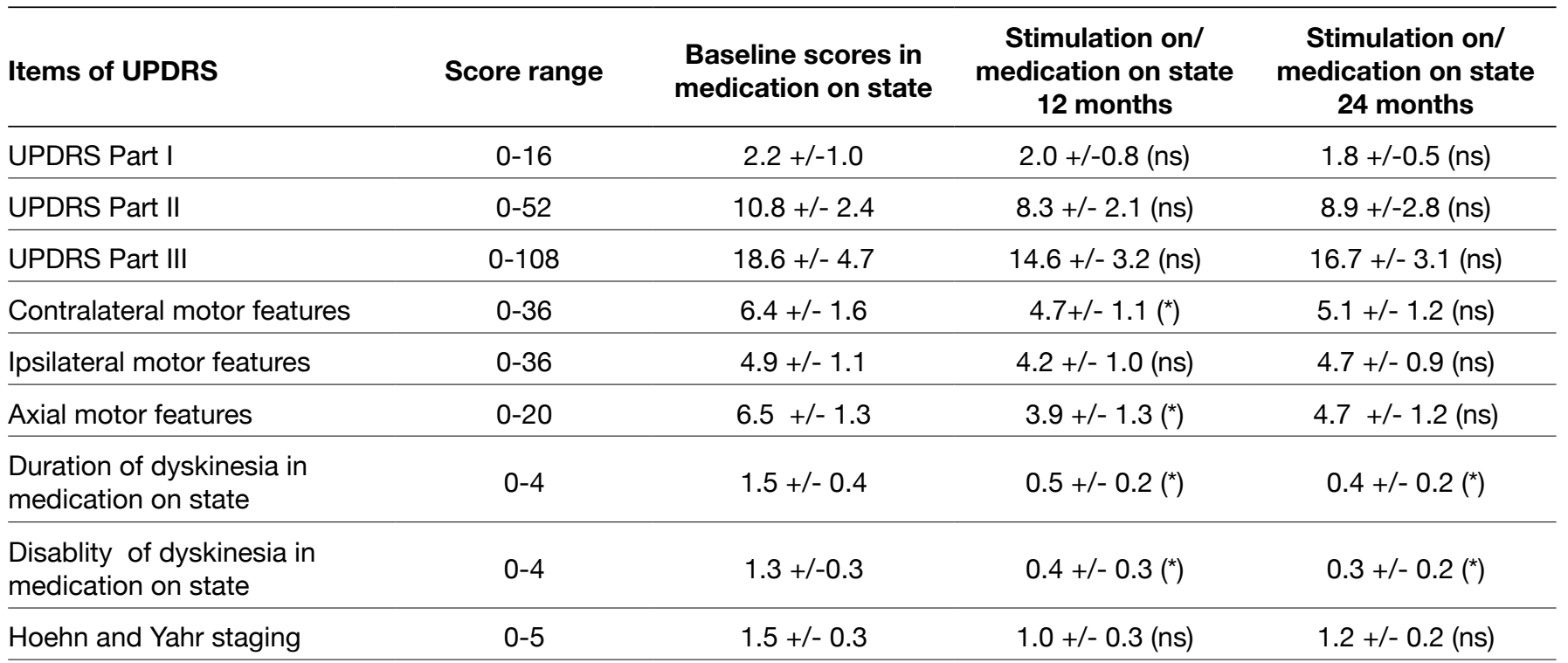

ADL: Activities of daily living, STN: Subthalamic nucleus, UPDRS: Unified Parkinson's Disease Rating Scale, DBS: Deep brain stimulation, LID: Levodopa-induced dyskinesia, (*): Statistical significance, ns: Not significant. 
UPDRS score was 33, which was comparable to preoperative motor UPDRS scores reported by other authors $(4,26)$, although other authors utilized unilateral STN DBS in more advanced PD $(22,23)$. Bilateral STN DBS has been reported to provide a 30 to $60 \%$ improvement in the total motor UPDRS score $(12,17,20,21,28)$. In a preliminary study reported by Verhagen in 25 patients with a 4-month follow-up unilateral STN DBS reduced the UPDRS motor score by $31 \%$ (24). Similar results were observed by Samii et al, who scheduled patients with advanced PD for staged bilateral STN DBS (19). Unilateral STN DBS resulted in a $25 \%$ improvement of the motor UPDRS score with a further $53 \%$ improvement when an additional DBS lead was implanted (19). Similar observations have been made by Kumar et al., who compared unilateral stimulation to bilateral STN stimulation in 10 patients by turning off one of the internal pulse generators (5). Unilateral STN DBS resulted in a $23 \%$ improvement of the motor UPDRS scores, with an additional $53 \%$ improvement of scores using bilateral STN DBS (5). It seems that bilateral STN DBS is more effective than unilateral STN DBS in improving PD symptoms, but on the other hand it involves additional side effects and costs $(5,19,21,28)$.

Unilateral STN DBS results in PD symptom improvement which is most visible on the contralateral side to the stimulated STN. The reduction of contralateral motor scores can range from 46 up to $75 \%$, according to reports of various authors $(2,4,5,8,17,19,22,23)$. In our series, contralateral motor UPDRS scores were improved by $49 \%$ at 2 -year follow-up. Similar results were obtained by Kumar et al. and Slowinski et al. $(5,22)$. The effects of unilateral STN DBS are not primarily restricted to the contralateral side, however most authors also report benefits in the ipsilateral and axial features (although the improvements tend to be only temporary $(22,23,26)$. In our series, axial motor features decreased by $18 \%$ at 2 years postoperatively in the off state. According to other authors, the improvement of axial motor features ranges between 10 and $36 \%$ in the off state $(2,4,5,8,17,19,22,23)$. Axial improvement seen after unilateral STN stimulation can be explained by restoration of excitability to the ipsilateral premotor cortex $(7,11)$. The STN projects predominantly to the ipsilateral globus pallidus internus GPi $(7,11)$. The pallidofugal fibers innervate the ventral anterior, ventral posterior and the centromedian nucleus of the thalamus. Approximately $20 \%$ of pallidofugal fibers cross the midline in the posterior section of the supracommissural decussation, and reach the same nuclei in the contralateral thalamus. In this situation, unilateral STN stimulation can affect the premotor cortex bilaterally (7). Unilateral STN stimulation may influence axial musculature in another way by descending pathways that connect to bilateral spinal motor neurons. STN projects not only directly to the GPi and to the substantia nigra pars reticulata SNr, but also to the ipsilateral pedunculopontine nucleus PPN $(7,11)$. Furthermore, the ipsilateral PPN neurons project to the contralateral PPN and to the reticular formation of the brainstem which, in turn, project bilaterally to the spinal cord. These abundant ascending and descending STN connections may explain the stimulation effect on axial symptoms as well ipsilateral motor features (13). Moreover, unilateral STN DBS may have antidromic effects on the cortical projection which can be bilateral. In our series, ipsilateral motor features were improved by $12 \%$ at 1 year. During the subsequent visit, at 2 years postoperatively, the unilateral effect was lost. The improvement of ipsilateral motor symptoms reported by other authors varied between 17 and 27\% $(23,26)$.

Unilateral STN DBS effectively reduced LID. Among our patients, the duration and disability of LID were reduced by $73 \%$ and $77 \%$ at 2 years, respectively. The outcomes are comparable to those reported by other investigators $(8,17,19,22,23)$.

The issue of second-side STN DBS surgery among the reported series is inconsistent and may be potentially associated with the maintenance or reduction of the daily levodopa dose during the follow-up period in unilateral STN DBS patients, as well as with the stage of PD at the time of the first surgery. Some authors applied unilateral STN DBS to patients with highly asymmetric PD $(4,26)$, while others operated on patients with advanced PD $(8,22)$. Nevertheless, ipsilateral deterioration of motor features was the reason for considering second-side STN DBS surgery in some series. In the study by $\mathrm{Kim}$ et al., all patients followed for up to 2 years considered second-side surgery, though it needs to be noted that these patients had highly asymmetric PD before their unilateral STN surgery (4). On the other hand, in the study by Slowinski et al. only five patients out of 16 individuals with symmetrical disease prior to surgery considered second-side surgery during the last follow-up visit (22). A similar observation is reported in the study by Germano et al. with a 12-month follow-up (2). As reported by those authors, only 2 patients out of 12 considered second-side surgery, even though all had advanced PD at the time of the unilateral STN DBS (mean $\mathrm{H} / \mathrm{Y}$ stage 3 in the off state) (2). Germano et al. concluded that sufficient improvement could be obtained with unilateral STN DBS in many patients with advanced PD, thereby avoiding the costs and risks associated with the second procedure (2). In the study by Linazasoro et al. involving a group of 8 patients who were scheduled for bilateral STN DBS with advanced PD but for various reasons received a unilateral implant, unilateral STN DBS provided good control of PD symptoms in 5 patients in the follow-up period varying from 6 to 16 months (8). The results, according to the authors, were quite unexpected and could be partially explained by the placebo effect that may account for $30 \%$ in PD patients and may persist for up to 18 months (3). In our series, only 4 patients considered second-side surgery at 2 years follow-up. This is attributable to a smaller reduction of the daily levodopa dose which - in our patients - was reduced only by $10 \%$ at 2 years, and the less advanced stage of PD preoperatively (mean UPDRS part III score 33). The decision whether to avoid the second surgery is, in our opinion, determined by maintaining a stable levodopa dose. For unilateral STN DBS we selected patients with asymmetric PD with mild to moderate LID. We did not observe any deterioration of LID after unilateral STN DBS, and there was no need to reduce levodopa after surgery. An opposite situation may prevail in patients with advanced PD undergoing unilateral STN DBS (5). Stimulation and levodopa may exacerbate contralateral dyskinesia which necessitates a reduction of the daily levodopa dose, which in turn fails to 
control ipsilateral off symptoms in the off state. This can be the reason why some patients with advanced PD after unilateral STN DBS require earlier second-side surgery (5).

Still, unilateral STN stimulation remains a valuable therapeutic option because of its safety and effectiveness. As observed by Slowinski et al., unilateral STN DBS may deliver a satisfactory clinical improvement for an unspecified period of time for many patients with a predominantly unilateral disease (22). A staged STN DBS may be even a more reasonable and arguably a safer approach, particularly for older patients (22). This belief is also supported by Linazasoro et al. who concluded that bilateral STN DBS approach is too aggressive in older PD patients, especially those over 70 years of age (8).

Bilateral simultaneous STN DBS can be associated with an increased risk of side effects including an increased risk of postoperative confusion, dysartria, dysphasia, and challenged attention and concentration $(10,15,18,21,29)$. Reports on the cognitive impairment of bilateral STN DBS have been quite inconsistent. In some series authors have observed no change - or even an improvement in cognitive domains (15). Other authors have reported a significant exacerbation of working memory, speed and mental processing, set switching, and verbal fluency $(18,29)$. These cognitive side effects have been encountered predominantly among older patients, over 69 years old $(21,28)$. Specific neuropsychological tests involving patients who underwent bilateral STN DBS have revealed attention and concentration deficits, and difficulties in verbal learning, naming, and fluency $(10,15)$. On the other hand, no cognitive deficits have been observed clinically after unilateral STN DBS $(22,26)$. The study by Slowinski et al., based on a battery of neuropsychological tests, revealed an improvement in social functioning, no significant postoperative changes in auditory-verbal attention, generating naming for semantic categories, learning, memory, and mood ratings (22). Other authors have also demonstrated the absence of cognitive deficits after unilateral STN DBS $(2,4,5,8,17,19,22,23)$.

Our observations support the belief that in PD patients unilateral STN DBS may provide satisfactory results. Even if the patients require a second-side STN DBS, a prolonged interval between consecutive surgical procedures may be an advantage $(2,8,22)$.

\section{CONCLUSIONS}

1) Unilateral STN DBS remains an efficient treatment option for PD patients. The stimulation effect was seen in the off state with a reduction of motor scores by $31 \%$ and $29 \%$ at 1 and 2 years after surgery.

2) Unilateral STN DBS successfully reduces LID disability by $69 \%$ and $77 \%$ at 1 , and 2 years after surgery, respectively.

3) The incidence rate of all complications (procedural and hardware-related) in the present series was $15.6 \%$. Two patients with procedural complications made full recovery, and 3 hardware related complications were managed surgically without any further complications.

\section{- REFERENCES}

1. Defer $G L$, Widner $H$, Marié RM, Rémy $P$, Levivier $M$ : Core assessment program for surgical interventional therapies in Parkinson's disease (CAPSIT-PD). Mov Disord 14(4):572-584, 1999

2. Germano IM, Gracies JM, Weisz DJ, Tse W, Koller WC, Olanow CW: Unilateral stimulation of the subthalamic nucleus in Parkinson disease: A double-blind 12-month evaluation study. J Neurosurg 101(1):36-42, 2004

3. Goetz C, Leurgans S, Raman M: Placebo-associated improvements in motor function: Comparison of subjective and objective sections of the UPDRS in early Parkinson's disease. Mov Disord 17:283-288, 2002

4. Kim HJ, Paek SH, Kim JY, Lee JY, Lim YH, Kim DG, Jeon BS: Two-year follow-up on the effect of unilateral subthalamic deep brain stimulation in highly asymmetric Parkinson's disease. Mov Disord 24(3):329-335, 2009

5. Kumar R, Lozano AM, Sime E, Halket E, Lang AE: Comparative effects of unilateral and bilateral subthalamic nucleus deep brain stimulation. Neurology 53 (3):561-566, 1999

6. Levin BE, Tomer R, Rey GJ: Cognitive impairments in Parkinson's disease. Neurol Clin 10(2):471-485, 1992

7. Levy R, Hazrati LN, Herrero MT, Vila M, Hassani OK, Mouroux M, Ruberg M, Asensi H, Agid Y, Féger J, Obeso JA, Parent A, Hirsch EC: Re-evaluation of the functional anatomy of the basal ganglia in normal and Parkinsonian states. Neuroscience 76(2):335-343, 1997

8. Linazasoro $\mathrm{G}$, Van Blercom N, Lasa A: Unilateral subthalamic deep brain stimulation in advanced Parkinson's disease. Mov Disord 18(6):713-716, 2003

9. Merims D, Freedman M: Cognitive and behavioral impairment in Parkinson's disease. Int Rev Psychiatr 20(4):364-373, 2008

10. Morrison CE, Borod JC, Perrine K, Beric A, Brin MF, Rezai A, Kelly P, Sterio D, Germano I, Weisz D, Olanow CW: Neuropsychological functioning following bilateral subthalamic nucleus stimulation in Parkinson's disease. Arch Clin Neuropsychol 19(2):165-181, 2004

11. Nakano K: Neuronal circuits and topographic organization of the basal ganglia and related regions. Brain Dev 22 Suppl 1:516,2000

12. Ostergaard K, Sunde N, Dupont E: Effects of bilateral stimulation of the subthalamic nucleus in patients with severe Parkinson's disease and motor fluctuations. Mov Disord 17(4): 693-700, 2002

13. Pahapill PA, Lozano AM: The pedunculopontine nucleus and Parkinson's disease. Brain 123(Pt 9): 1767-1783, 2000

14. Perez-Lloret S, Rey MV, Fabre N, Ory F, Spampinato U, BrefelCourbon C, Montastruc JL, Rascol O: Prevalence and pharmacological factors associated with impulse-control disorder symptoms in patients with Parkinson disease. Clin Neuropharm 35(6):261-265, 2012

15. Pillon B: Neuropsychological assessment for management of patients with deep brain stimulation. Mov Disord 17 Suppl 3: 116-122, 2002 
16. Riedel O, Klotsche J, Spottke A, Deuschl G, Förstl H, Henn F, Heuser I, Oertel W, Reichmann H, Riederer P,Trenkwalder C, Dodel R, Wittchen HU: Frequency of dementia, depression, and other neuropsychiatric symptoms in 1,449 outpatients with Parkinson's disease. J Neurol 257(7):1073-1082, 2010

17. Romito LM, Scerrati M, Contarino MF, Bentivoglio AR, Tonali P, Albanese A: Long term follow-up of subthalamic nucleus stimulation in Parkinson's disease. Neurology 58(10):15461550, 2002

18. Saint-Cyr JA, Trépanier LL, Kumar R, Lozano AM, Lang AE: Neuropsychological consequences of chronic bilateral stimulation of the subthalamic nucleus in Parkinson's disease. Brain 123:2091-108, 2000

19. Samii A, Kelly VE, Slimp JC, Shumway-Cook A, Goodkin $\mathrm{R}$ : Staged unilateral versus bilateral subthalamic nucleus stimulator implantation in Parkinson disease. Mov Disord 22(10):1476-1481, 2007

20. Schüpbach WM, Maltête D, Houeto JL, du Montcel ST, Mallet L, Welter ML, Gargiulo M, Béhar C, Bonnet AM, Czernecki V, Pidoux B, Navarro S, Dormont D, Cornu P, Agid Y: Neurosurgery at an earlier stage of Parkinson disease: A randomized, controlled trial. Neurology 68(4):267-271, 2007

21. Simuni T, Jaggi JL, Mulholland H, Hurtig HI, Colcher A, Siderowf AD, Ravina B, Skolnick BE, Goldstein R, Stern $\mathrm{MB}$, Baltuch $\mathrm{GH}$ : Bilateral stimulation of the subthalamic nucleus in patients with Parkinson's disease: A study of efficacy and safety. J Neurosurg 96(4):666-672, 2002

22. Slowinski JL, Putzke JD, Uitti RJ, Lucas JA, Turk MF, Kall BA, Wharen RE: Unilateral deep brain stimulation of the subthalamic nucleus for Parkinson disease. J Neurosurg 106(4):626-632, 2007
23. Tabbal SD, Ushe M, Mink JW, Revilla FJ, Wernle AR, Hong M, Karimi M, Perlmutter JS: Unilateral subthalamic nucleus stimulation has a measurable ipsilateral effect on rigidity and bradykinesia in parkinson disease. Exp Neurol 211(1):234242, 2008

24. Verhagen L, Arzbaecher J, Sierens D, Myre B, Verwey NA, Leurgans S, Bakay Roy A: Unilateral deep brain stimulation of the subthalamic nucleus: A valuable alternative? Neurology 60(5 Supplement 1): A119, Ch 11, 2003

25. Volkmann J, Herzog J, Kopper F, Deuschl G: Introduction to the programming of deep brain stimulators. Mov Disord 17 Suppl 3:S181-187, 2002

26. Walker HC, Watts RL, Guthrie S, Wang D, Guthrie BL: Bilateral effects of unilateral subthalamic deep brain stimulation on Parkinson's disease at 1 year. Neurosurgery 65(2):302-309, 2009

27. Weiss HD, Marsh L: Impulse control disorders and compulsive behaviors associated with dopaminergic therapies in Parkinson disease. Neurol Clin Pract 2(4):267-274, 2012

28. Welter ML, Houeto JL, Tezenas du Montcel S, Mesnage V, Bonnet AM, Pillon B, Arnulf I, Pidoux B, Dormont D, Cornu $\mathrm{P}$, Agid Y: Clinical predictive factors of subthalamic stimulation in Parkinson's disease. Brain 125(3):575-583, 2002

29. Woods SP, Fields JA, Troster Al: Neuropsychological sequelae of subthalamic nucleus deep brain stimulation in Parkinson's disease: A critical review. Neuropsychol Rev 12(2):111-126, 2002 